\title{
Recent Advances in Chromatography for the Efficient Profiling of Crude Extracts and Isolation of Natural Products
}

\author{
Jean-Luc Wolfender
}

School of Pharmaceutical Sciences, EPGL, University of Geneva, University of Lausanne, 30, quai Ernest-Ansermet, CH-1211Geneva, Switzerland; E-mail: jean-luc.wolfender@unige.ch

The large chemical space occupied by natural products (NPs) is directly linked to a high variability of their intrinsic physicochemical properties that render their separation, detection and characterization challenging. The analysis of NPs in complex crude extracts requires efficient separation methods. In this respect, high-performance liquid chromatography (HPLC) has been recognized since the early 1980s as the most versatile technique for their profiling directly in crude mixtures without the need for complex sample preparation [1]. HPLC has greatly developed through the years in terms of convenience, speed, choice of column stationary phases, high sensitivity, applicability to a broad variety of sample matrices, and ability to hyphenate the chromatographic methods to spectroscopic detectors. The latest developments of HPLC, including the recent introduction of very $\mathrm{pH}$-stable phases, sub-2- $\mu \mathrm{m}$ particles, monolith and fused core columns, have considerably improved the performances of HPLC. In particular in phytochemical analysis, the recent introduction of Ultra High Pressure Liquid Chromatography (UHPLC) systems operating at very high pressures and using sub-2 $\mu$ m packing columns have allowed a remarkable decrease in analysis time and increase in peak capacity, sensitivity and reproducibility compared to conventional HPLC [2]. Such developments have a significant impact on the quality of data that can be obtained for metabolite profiling, dereplication studies and metabolomics when efficient spectroscopic detectors such as time-of-flight mass spectrometers (TOF)-MS are hyphenated to UHPLC. On the other hand, a good mastery of the chromatography parameters at the analytical scale enables efficient gradient transfer to the semi-preparative or the preparative scale based on chromatographic calculation. This efficient upscale allows high loading of crude mixture either for rapid at-line biological profiling of crude extracts with sensitive assay and/or de novo structure determination with microflow NMR methods with microgram of NPs. These developments, as well as other new trends in chromatography such as the renewed interest for superfluid critical fluid separation, the use of two dimensional LC or the hyphenation with additional separation efficiency in the gas phase provided by ion mobility in LC-MS hyphenation for the deconvolution of NPs in complex crude plant extracts will be discussed.

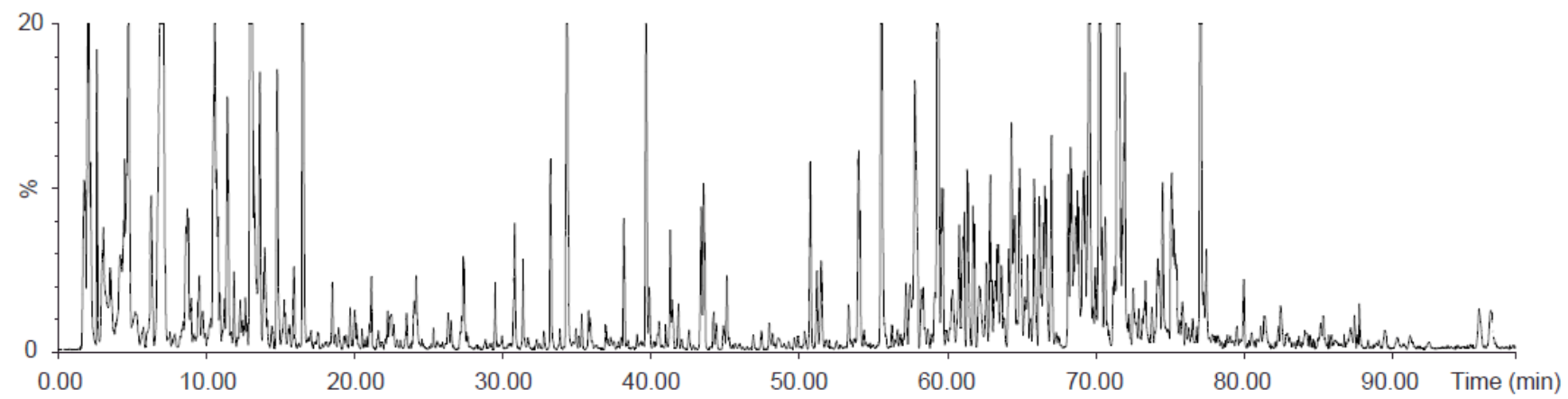

Example of a high resolution UHPLC-TOF-MS profiling of the crude leaf extract of A. thaliana.

\section{REFERENECES}

[1] Wolfender JL. HPLC in natural product analysis: the detection issue. Planta Med 2009; 75: 719-34.

[2] Eugster P, Guillarme D, Rudaz S, Veuthey JL, Carrupt PA, Wolfender JL. Ultra high pressure liquid chromatography for crude plant extract profiling. J AOAC Int 2011; 94: 51-70. 